\title{
Ventricular orexin-A (hypocretin-I) levels correlate with rapid-eye-movement sleep without atonia in Parkinson's disease
}

This article was published in the following Dove Press journal:

Nature and Science of Sleep

14 June 2013

Number of times this article has been viewed

\author{
Agathe Bridoux ${ }^{1,2}$ \\ Stephane Moutereau ${ }^{3}$ \\ Ala Covali-Noroc' \\ Laurent Margarit' \\ Stephane Palfi ${ }^{4}$ \\ Jean-Paul Nguyen ${ }^{5}$ \\ Jean-Pascal Lefaucheur ${ }^{1,2}$ \\ Pierre Césaro ${ }^{6}$ \\ Marie-Pia d'Ortho' \\ Xavier Drouot ${ }^{1,2}$ \\ 'Service de Physiologie, Groupe \\ Henri Mondor, ${ }^{2}$ Faculté de Médecine, \\ Université Paris Est Créteil, ${ }^{3}$ Service \\ de Biochimie, Groupe Henri Mondor, \\ ${ }^{4}$ UF Neurochirurgie Fonctionnelle, \\ Groupe Henri Mondor, Créteil, \\ France; ${ }^{5}$ Service de Neurochirurgie, \\ Hôpital Nord Laënnec, Nantes, \\ France; ${ }^{6}$ Service de Neurologie, \\ Groupe Henri Mondor, Créteil, \\ France; ${ }^{7}$ Service de Physiologie, \\ Groupe Bichat - Claude Bernard, \\ Paris, France
}

Objective: Patients with Parkinson's disease frequently complain of sleep disturbances and loss of muscle atonia during rapid-eye-movement (REM) sleep is not rare. The orexin-A (hypocretin-1) hypothalamic system plays a central role in controlling REM sleep. Loss of orexin neurons results in narcolepsy-cataplexy, a condition characterized by diurnal sleepiness and REM sleep without atonia. Alterations in the orexin-A system have been also documented in Parkinson's disease, but whether these alterations have clinical consequences remains unknown.

Methods: Here, we measured orexin-A levels in ventricular cerebrospinal fluid from eight patients with Parkinson's disease (four males and four females) who underwent ventriculography during deep brain-stimulation surgery and performed full-night polysomnography before surgery.

Results: Our results showed a positive correlation between orexin-A levels and REM sleep without muscle atonia.

Conclusion: Our results suggest that high levels of orexin-A in Parkinson's disease may be associated with loss of REM muscle atonia.

Keywords: Parkinson, orexin-A, ventricular CSF, REM atonia

\section{Introduction}

Sleep disorders and diurnal sleepiness are frequent in Parkinson's disease. ${ }^{1}$ Polysomnography studies have shown rapid-eye-movement (REM) sleep dysregulation with REM sleep onset on diurnal naps ${ }^{2,3}$ and loss of muscle atonia, ${ }^{4}$ which is defined as "excessive amount of sustained or intermittent elevation of submental electromyographic tone or excessive phasic submental or limb electromyographic twitching." ${ }^{4}$ Loss of REM atonia is a core phenomenon of REM sleep behavioral disorder (RBD), ${ }^{5}$ a condition characterized by vigorous and injurious motor behaviors during REM sleep. ${ }^{6}$

The orexin system plays a key role in REM sleep regulation. The loss of orexin neurons results in narcolepsy-cataplexy, a condition characterized by severe diurnal sleepiness, REM sleep onset during daytime naps, and cataplexies. REM sleep without atonia (RSWA) and RBD have also been reported to occur in narcolepsy. ${ }^{7}$

Orexin neurotransmission has been shown to be altered in Parkinson's disease (PD) ${ }^{8-10}$ However, polysomnographic data were not available in these postmortem studies. Furthermore, we showed that ventricular orexin levels were not correlated with mean sleep latency, suggesting that orexin deficit might not be involved in daytime sleepiness in PD. ${ }^{11}$ Thus, the clinical expression of orexin deficit in PD remains unknown. ${ }^{12}$
Correspondence: Agathe Bridoux Service de Physiologie Explorations Fonctionnelles, AP-HP, Groupe Hospitalier Henri Mondor - Albert Chenevier, Créteil F-940 I0, France Tel +33 । 498 I 4670

$\mathrm{Fax}+33$ । 498 8। 4660

Email agathe.bridoux@hmn.aphp.fr 
The objective of this study was to determine whether orexin-A levels in ventricular cerebrospinal fluid (CSF) from PD patients were correlated with REM sleep without atonia.

\section{Patients and methods Patients}

Consecutive patients with idiopathic $\mathrm{PD}^{13}$ who were scheduled for deep brain-stimulation neurosurgery were invited to participate in the study, irrespective of whether they had sleep complaints. We did not include patients taking medications known to affect sleep or REM atonia or patients with sleep apnea syndrome defined as an apnea/hypopnea index greater than ten per hour of sleep. Disease severity was assessed based on the motor subscore of the Unified Parkinson's Disease Rating Scale III after overnight withdrawal of dopaminergic treatment. The study was approved by the appropriate ethics committee, and informed consent was obtained from all patients prior to study inclusion.

\section{Polysomnography}

Sleep recordings were obtained during attended polysomnography 1 week before the scheduled date of neurosurgery. Polysomnography included electroencephalogram recordings (C4-A1, C3-A2, and O1-O2 leads), two electrooculograms, one submental electromyogram (EMG), and bilateral anterior tibialis EMGs. Also recorded were nasal pressure, oral airflow, thoracic and abdominal movements, and pulse oximetry. Video recordings were not obtained. Patients took their usual PD medications and were free to go to sleep when they wanted to.

Sleep stages were scored by two sleep specialists who had extensive experience with recordings in PD patients ( $\mathrm{AB}$ and $\mathrm{XD}$ ), according to the modified American Academy of Sleep Medicine criteria, ${ }^{14}$ with allowance for RSWA. RSWA was scored as previously described by Lapierre and Montplaisir. ${ }^{16}$ Tonic chin-muscle activity during REM sleep was defined as chin EMG amplitude greater than twice the amplitude measured during a baseline EMG signal for atonia. Phasic EMG events were defined as any burst of activity lasting $0.3-5$ seconds and having amplitude greater than four times the baseline EMG signal. Tonic and phasic EMG activities were scored from the submental EMG signal per 3-second mini-epochs of REM sleep containing or not containing "any" EMG activity (tonic, phasic, or a combination of both EMG activities). ${ }^{17}$ We then reported loss of REM atonia as the percentage of REM sleep mini-epochs with "any" EMG activity. Tonic and phasic EMG increases associated with respiratory arousals were excluded from the analysis.

\section{Collection of ventricular cerebrospinal fluid}

Ventriculography performed to assist in the proper placement of an electrode in the subthalamic nucleus require the removal of CSF just before the injection of the contrast agent into the ventricle. In each of our patients, $3 \mathrm{~mL}$ of ventricular CSF was immediately frozen and stored at $-80^{\circ} \mathrm{C}$ until use for the orexin-A assay. ${ }^{8}$

\section{Orexin-A assay}

Orexin-A (hypocretin-1) was measured in crude CSF using a commercially available radioimmunoassay kit (Phoenix Pharmaceuticals, Belmont, CA, USA). The detection limit was $50 \mathrm{pg} / \mathrm{mL}$, and intra-assay variability was $5 \%$. Each CSF sample was tested in duplicate.

\section{Statistics}

Correlations between orexin-A levels and sleep parameters were evaluated using Spearman's rank-correlation test and the nonparametric Mann-Whitney test. $P$-values smaller than 0.05 were considered significant.

\section{Results \\ Patients}

We studied eight consecutive patients - four males and four females - whose clinical features are reported in Table 1 . In all eight patients, surgery was performed under regional anesthesia between $10 \mathrm{am}$ and $12 \mathrm{am}$.

No patients or bed partners reported violent movements during sleep, although the spouse of patient $\# 7$ reported limb and body jerking.

\section{Correlation between orexin-A levels and nighttime sleep parameters}

Orexin-A levels did not correlate with total sleep time ( $P=0.23)$, REM duration $(P=0.45)$, or REM latency $(P=0.66)$. In contrast, orexin-A levels showed a significant positive correlation with the percentage of REM epochs without atonia, ie, percentage of 3-second mini-epochs classified as "any" ( $\rho=0.83, P=0.027)$ (Figure 1A).

As previously described, ${ }^{6,17}$ RSWA is considered clinically significant when any submental EMG activity was present for more than $18 \%$ of the total REM sleep duration. According to this criterion, four of our patients had RSWA (\#2, \#3, \#4, and \#7). These four patients had higher median 
Table I Nighttime sleep parameters

\begin{tabular}{|c|c|c|c|c|c|c|c|c|}
\hline & \#I, F & $\# 2, \mathrm{~F}$ & $\# 3, \mathrm{~F}$ & $\# 4, F$ & $\# 5, \mathrm{M}$ & $\# 6, M$ & $\# 7, \mathrm{M}$ & $\# 8, M$ \\
\hline Age, years & 62 & 66 & 52 & 52 & 69 & 48 & 63 & 54 \\
\hline Disease duration (years) & 13 & 18 & 15 & 8 & 7 & 12 & 18 & 11 \\
\hline Sleep latency (minutes) & 45 & 10 & 46.5 & 16.7 & 66.5 & 10.5 & 24 & 20 \\
\hline Sleep efficiency (\%) & 89 & 78 & 50.3 & 80.2 & 45.3 & 77 & 84 & 80 \\
\hline TST (minutes) & 413 & 375 & 311 & 385 & 269 & 360 & 467 & 346 \\
\hline Stage N2 (minutes) & 41 & 60 & 44 & 70 & 40 & 49 & 43 & 47 \\
\hline Stage N3 (minutes) & 111 & 49 & 67 & 32 & 83 & 82 & 110 & 104 \\
\hline REM (\%) & 24 & 20 & 21 & 7 & 17 & 26 & 28 & 14 \\
\hline REM latency (minutes) & 115 & 65 & 112 & 95 & 128 & 216 & 109 & 64 \\
\hline 3-second REM epochs with any activity (\%) & 6 & 37 & 19 & 43 & 5 & 6 & 33 & 4 \\
\hline $\mathrm{AHI}$ (events/hour) & 7 & 2 & 8 & 3 & 7 & 10 & 9 & 10 \\
\hline PLMs (events/hour) & 0 & 2 & 0 & 5 & 0 & 4 & 0 & 17 \\
\hline Fragmentation (events/hour) & 20 & 27 & 21 & 28 & 18 & 9 & 11 & 12 \\
\hline Orexin-A (pg/mL) & 50 & 240 & 200 & 230 & 138 & 156 & 450 & 39 \\
\hline
\end{tabular}

Notes: Sleep latency was nighttime sleep latency of the first N2 epoch; sleep stage N3 is slow-wave sleep; any activity/REM was calculated as percentage of 3-second REM epochs with "any" activity.

Abbreviations: TST, total sleep time; REM, rapid eye movement; AHI, apnea/hypopnea index; PLMs, periodic limb movements; F, female; M, male.

(interquartile range) orexin-A levels than the four patients with REM sleep atonia (235 pg/mL [215-345] vs $94 \mathrm{pg} / \mathrm{mL}$ [44-147]; Mann-Whitney test $P=0.036$, Figure 1B). No other sleep parameters differed significantly between these two groups of patients.

\section{Discussion}

Our results show a significant relationship between ventricular CSF orexin-A levels and REM sleep characteristics in patients with PD. Despite the small number of patients, we found a correlation between orexin-A levels and RSWA. Our results suggest that orexin-A signaling may be associated with loss of muscle atonia during REM sleep in patients with PD.

To our knowledge, this is the first study of potential correlations between ventricular CSF orexin-A levels and polysomnographic data in patients with PD. We undertook this study to investigate the physiological significance of altered orexin neurotransmission in $\mathrm{PD}$, which has generated controversy. ${ }^{12}$ The strength of our study is the use of ventricular CSF. A previous study in PD patients found that ventricular CSF orexin levels correlated with the number of orexinergic neurons. ${ }^{9}$ Furthermore, while a lumbar CSF study failed to report a significant correlation between orexin levels
A

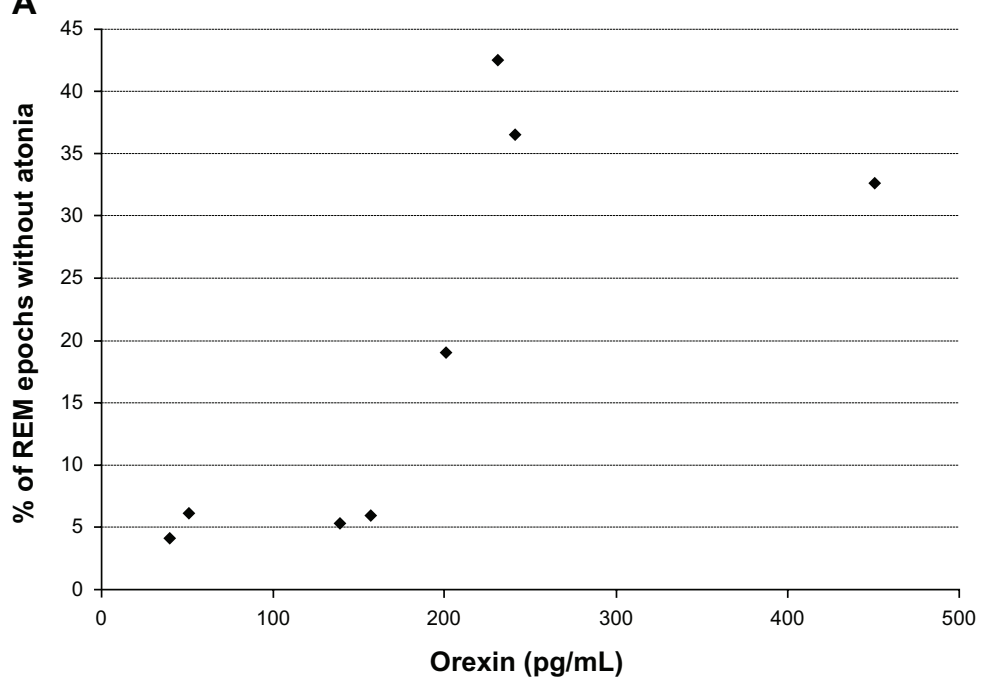

B

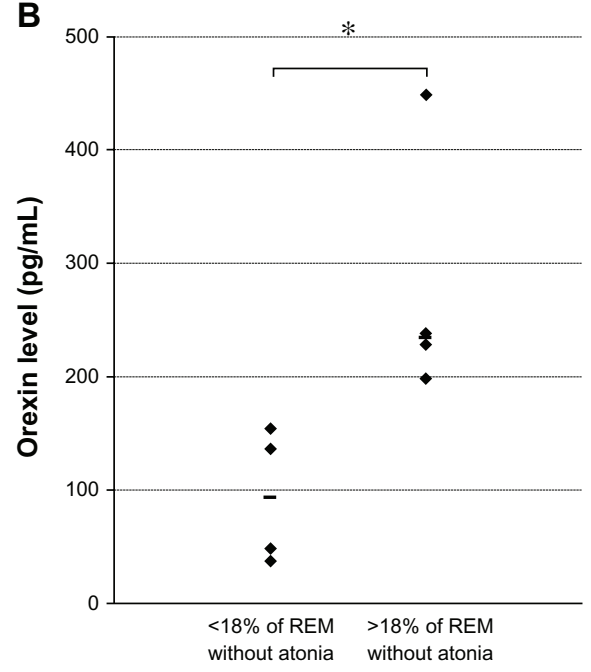

Figure I (A) Association between ventricular orexin-A levels $(\mathrm{pg} / \mathrm{mL})$ and loss of rapid-eye-movement $(\mathrm{REM})$ atonia. (B) Orexin-A levels ( $\mathrm{pg} / \mathrm{mL})$ based on the percentage of REM without atonia.

Notes: (A) Plot of the percentage of REM sleep mini-epochs without atonia (using "any" electromyographic activity) and orexin-A levels for each patient. (B) Orexin-A levels in patients with less than $18 \%(n=4$, left) and in patients with more than $18 \%(n=4$, right) of REM epochs without atonia. Horizontal black bars represent median values for each group. ${ }^{*} P<0.05$. 
and disease severity in a large group of $62 \mathrm{PD}$ patients, ${ }^{18}$ a significant correlation was reported with ventricular samples. $^{8}$

The main limitations of our study are the small number of patients and the absence of control subjects. We excluded patients taking sleep-altering medications and EMG activities concurrent with respiratory arousals, ${ }^{19}$ which may have further limited bias in our study.

In our study, PD patients with higher ventricular CSF orexin levels exhibited larger numbers of RSWA epochs than did patients with lower orexin levels, suggesting that preserved orexin drive may be involved in loss of REM atonia. These results are congruent with experiments demonstrating that orexin neurons are strongly involved in muscle-tone control. Orexin neurons exert a facilitating influence on muscle tone, both directly via their projections on trigeminal motor neurons ${ }^{20}$ and spinal motor neurons ${ }^{21}$ and indirectly via the locus coeruleus neurons. ${ }^{22}$ Orexin injections into the locus coeruleus increase muscle tone ${ }^{23}$ and interrupt pedunculopontine nucleus-induced muscle atonia. ${ }^{24}$ However, regulation of muscle atonia relies on complex relationships and interactions between the orexin system, the locus coeruleus (in which orexin facilitates muscle activity), and the pontine inhibitory area (in which orexin inhibits muscle activity).

Our results contrast with those obtained in narcolepsycataplexy, in which orexin levels negatively correlated with RSWA. ${ }^{25}$ However, narcolepsy-cataplexy and PD are two different diseases, and the process leading to orexinergic neuron loss is likely different. Orexin neurons are the primary target of an acute and specific neuronal aggression in narcolepsy, leading to a $90 \%$ reduction in the orexin neuron pool. ${ }^{26}$ In contrast, PD is a chronic, slowly progressive neurodegenerative disease, affecting primarily dopaminergic neurons, with a process not fully understood, leading to partial $(23 \%-60 \%)$ orexin neuron loss. ${ }^{10}$ As an illustration, no cataplexy has been reported in PD patients, even in patients with undetectable orexin levels. ${ }^{8,12}$

Our results are also in contrast with a recent study that found similar orexin lumbar levels in five RBD patients. ${ }^{27}$ However, these patients had idiopathic RBD and no sign of PD.

\section{Conclusion}

The loss of REM atonia in patients with higher orexin levels illustrates the complexity of the symptoms induced by altered orexin neurotransmission in PD. ${ }^{28}$ Experimental trials of orexin antagonists could be of interest in the transgenic mouse model of RSWA.

\section{Disclosure}

The authors report no conflicts of interest in this work.

\section{References}

1. Garcia-Borreguero D, Larrosa O, Bravo M. Parkinson's disease and sleep. Sleep Med Rev. 2003;7(2):115-129.

2. Arnulf I, Konofal E, Merino-Andreu M, et al. Parkinson's disease and sleepiness: an integral part of PD. Neurology. 2002;58(7):1019-1024.

3. Baumann C, Ferini-Strambi L, Waldvogel D, Werth E, Bassetti CL. Parkinsonism with excessive daytime sleepiness - a narcolepsy-like disorder? J Neurol. 2005;252(2):139-145.

4. Iranzo A, Santamaría J, Rye DB, et al. Characteristics of idiopathic REM sleep behavior disorder and that associated with MSA and PD. Neurology. 2005;65(2):247-252.

5. American Academy of Sleep Medicine. The International Classification of Sleep Disorders: Diagnostic and Coding Manual, 2nd ed. Darien (IL): American Academy of Sleep Medicine; 2005.

6. Gagnon JF, Bédard MA, Fantini ML, et al. REM sleep behavior disorder and REM sleep without atonia in Parkinson's disease. Neurology. 2002; 59(4):585-589

7. Dauvilliers Y, Arnulf I, Mignot E. Narcolepsy with cataplexy. Lancet. 2007;369(9560):499-511.

8. Drouot X, Moutereau S, Nguyen JP, et al. Low levels of ventricular CSF orexin/hypocretin in advanced PD. Neurology. 2003;61(4):540-543.

9. Fronczek R, Overeem S, Lee SY, et al. Hypocretin (orexin) loss in Parkinson's disease. Brain. 2007;130(Pt 6):1577-1585.

10. Thannickal TC, Lai YY, Siegel JM. Hypocretin (orexin) cell loss in Parkinson's disease. Brain. 2007;130(Pt 6):1586-1595.

11. Drouot X, Moutereau S, Lefaucheur JP, et al. Low level of ventricular CSF orexin-A is not associated with objective sleepiness in PD. Sleep Med. 2011;12(9):936-937.

12. Baumann CR, Scammell TE, Bassetti CL. Parkinson's disease, sleepiness and hypocretin/orexin. Brain. 2008;131(Pt 3):e91.

13. Hughes AJ, Daniel SE, Kilford L, Lees AJ. Accuracy of clinical diagnosis of idiopathic Parkinson's disease: a clinico-pathological study of 100 cases. J Neurol Neurosurg Psychiatry. 1992;55(3):181-184.

14. Silber MH, Ancoli-Israel S, Bonnet MH, et al. The visual scoring of sleep in adults. J Clin Sleep Med. 2007;3(2):121-131.

15. Consens FB, Chervin RD, Koeppe RA, et al. Validation of a polysomnographic score for REM sleep behavior disorder. Sleep. 2005;28(8): 993-997.

16. Lapierre O, Montplaisir J. Polysomnographic features of REM sleep behavior disorder: development of a scoring method. Neurology. 1992; 42(7):1371-1374.

17. Frauscher B, Iranzo A, Gaig C, et al. Normative EMG values during REM sleep for the diagnosis of REM sleep behavior disorder. Sleep. 2012;35(6):835-847.

18. Yasui K, Inoue Y, Kanbayashi T, Nomura T, Kusumi M, Nakashima K. CSF orexin levels of Parkinson's disease, dementia with Lewy bodies, progressive supranuclear palsy and corticobasal degeneration. J Neurol Sci. 2006;250(1-2):120-123.

19. Iranzo A, Santamaria J. Severe obstructive sleep apnea/hypopnea mimicking REM sleep behavior disorder. Sleep. 2005;28(2):203-206.

20. Peever JH, Lai YY, Siegel JM. Excitatory effects of hypocretin-1 (orexin-A) in the trigeminal motor nucleus are reversed by NMDA antagonism. J Neurophysiol. 2003;89(5):2591-2600.

21. Yamuy J, Fung SJ, Xi M, Chase MH. Hypocretinergic control of spinal cord motoneurons. J Neurosci. 2004;24(23):5336-5345.

22. Mileykovskiy BY, Kiyashchenko LI, Siegel JM. Muscle tone facilitation and inhibition after orexin-a (hypocretin-1) microinjections into the medial medulla. J Neurophysiol. 2002;87(5):2480-2489.

23. Kiyashchenko LI, Mileykovskiy BY, Lai YY, Siegel JM. Increased and decreased muscle tone with orexin (hypocretin) microinjections in the locus coeruleus and pontine inhibitory area. J Neurophysiol. 2001; 85(5):2008-2016. 
24. Takakusaki K, Takahashi K, Saitoh K, et al. Orexinergic projections to the cat midbrain mediate alternation of emotional behavioral states from locomotion to cataplexy. J Physiol. 2005;568(Pt 3):1003-1020.

25. Knudsen S, Gammeltoft S, Jennum PJ. Rapid eye movement sleep behaviour disorder in patients with narcolepsy is associated with hypocretin-1 deficiency. Brain. 2010;133(Pt 2):568-579.

26. Thannickal TC, Moore RY, Nienhuis R, et al. Reduced number of hypocretin neurons in human narcolepsy. Neuron. 2000;27(3):469-474.
27. Anderson KN, Vincent A, Smith IE, Shneerson JM. Cerebrospinal fluid hypocretin levels are normal in idiopathic REM sleep behaviour disorder. Eur J Neurol. 2010;17(8):1105-1107.

28. Fronczek R, Baumann CR, Lammers GJ, Bassetti CL, Overeem S. Hypocretin/orexin disturbances in neurological disorders. Sleep Med Rev. 2009;13(1):9-22.

\section{Publish your work in this journal}

Nature and Science of Sleep is an international, peer-reviewed, open access journal covering all aspects of sleep science and sleep medicine, including the neurophysiology and functions of sleep, the genetics of sleep, sleep and society, biological rhythms, dreaming, sleep disorders and therapy, and strategies to optimize healthy sleep. The journal welcomes
Dovepress

original research, clinical \& epidemiological studies, reviews \& evaluations, case reports and extended reports. The manuscript management system is completely online and includes a very quick and fair peerreview system, which is all easy to use. Visit http://www.dovepress.com/ testimonials.php to read real quotes from published authors.

Submit your manuscript here: http://www.dovepress.com/nature-and-science-of-sleep-journal 